\title{
Expresión de Ki67 y MUC-1 en el Adenocarcinoma No Especificado de otra Manera (NOS) de Glándulas Salivales: su Valor Pronóstico
}

\author{
Ki67 and MUC-1 Expression in Adenocarcinoma Not Otherwise \\ Specified (NOS) of Salivary Glands: Prognostic Value
}

\author{
María Elena Samar Romani'; Rodolfo Esteban Ávila Uliarte ${ }^{2}$; Pedro Emilio García Esst ${ }^{3}$; \\ Ismael Bernardo Fonseca Acosta ${ }^{4} \&$ Javier Elías Fernández Calderón ${ }^{5}$
}

\begin{abstract}
SAMAR, R. M. E.; ÁVILA, U. R. E.; GARCÍA, E. P. E.; FONSECA, A. I. B. \& FERNÁNDEZ, C. J. E. Expresión de Ki67 y MUC-1 en el adenocarcinoma no especificado de otra manera (nos) de glándulas salivales: su valor pronóstico. Int. J. Odontostomat., 14(3):407-416, 2020.

RESUMEN: El adenocarcinoma NOS (no especificado de otra manera) es un tumor salival sin patrón especial poco mencionado en la literatura; su diagnóstico es un desafío porque estructuralmente no se identifica con otros carcinomas salivales más definidos. Por otro lado, Ki67 es un marcador de proliferación celular que brinda información pronóstica de las neoplasias. En cuanto a la mucina humana transmembrana MUC-1 se sobre-expresa en las neoplasias malignas perdiendo su localización exclusivamente apical. Presentamos dos casos de adenocarcinoma NOS diagnosticados con H/E y correlacionamos la expresión de Ki67 y la localización y sobreexpresión de MUC-1 con su grado histológico y pronóstico. Cortes histológicos de dos adenocarcinomas NOS de parótida en mujeres de 62 y 63 años respectivamente se colorearon con $\mathrm{H} / \mathrm{E}$ e inmunomarcaron para Ki67 y MUC-1. En ambos tumores predominaban estructuras ductales, algunas quísticas, cordones celulares ramificados e islotes sólidos. Las formaciones glandulares presentaban células claras y algunas de aspecto oncocítico. Había importante atipia celular, comedonecrosis, invasión perineural, áreas hemorrágicas y compromiso de los márgenes quirúrgicos. La marcación nuclear con Ki67 fue importante; MUC-1 presentó una fuerte coloración en membranas y citoplasmas. Las dos lesiones se diagnosticaron como de alto grado de malignidad. Nuestros resultados demuestran que existe una importante proliferación marcada con Ki67 y una sobre-expresión de MUC-1 asociadas a atipia celular, infiltración perineural, necrosis y compromiso de márgenes quirúrgicos, factores asociados a un peor pronóstico. El reconocimiento de este tumor es trascendente para médicos y odontólogos ya que por la ausencia de rasgos distintivos que sí presentan otros carcinomas más específicos es fundamental el diagnóstico de exclusión.
\end{abstract}

PALABRAS CLAVE: parótida, adenocarcinoma NOS, Ki67, MUC-1, pronóstico.

\section{INTRODUCCIÓN}

Los tumores de las glándulas salivales son raros y muy heterogéneos, tanto por su estructura histológica como por sus particularidades clínicas. Menos del $1 \%$ de todos los tumores y 3 a $10 \%$ de los correspondientes a cabeza y cuello se localizan en las glándulas salivales (Carlson \& Schlieve, 2019). Dentro de ellos el adenocarcinoma no especificado de otra manera (adenocarcinoma not otherwise specified -adenocarcinoma NOS-), de escasa mención en la literatura, es un tumor sin patrón especial que nos induce a un desafío debido a que su diferenciación estructural no se corresponde con otros tipos de carcinomas salivales de origen epitelial más definidos.

\footnotetext{
${ }^{1}$ Departamento de Biología Bucal, Facultad de Odontología, Universidad Nacional de Córdoba, Argentina.

${ }^{2}$ Cátedra de Biología Celular, Histología y Embriología, Facultad de Ciencias Médicas, Universidad Nacional de Córdoba, Argentina.

${ }^{3}$ Médico Oncólogo Clínico. Instituto de Radioterapia Zunino, Fundación Marie Curie. Profesor Asistente. Cátedra de Biología Celular, Histología y Embriología, Facultad de Ciencias Médicas, Universidad Nacional de Córdoba, Argentina.

${ }^{4}$ Cátedra de Patología, Escuela Práctica, Facultad de Ciencias Médicas, Universidad Nacional de Córdoba, Argentina.

${ }^{5}$ Departamento de Biología Bucal, Facultad de Odontología, Universidad Nacional de Córdoba, Argentina.

Subsidio CONSOLIDAR 05/H575. Período 2018-2021. Secretaría de Ciencia y Tecnología. Universidad Nacional de Córdoba. Argentina.
} 
La Organización Mundial de la Salud (El-Naggar et al., 2017) describe el adenocarcinoma no especificado de otra manera (adenocarcinoma, NOS - ICD Code 8140/03, International Classification of Diseases for Oncology) como una serie de carcinomas salivales epiteliales con una disposición glandular y/o ductal con o sin formación de quistes.

Ellis \& Auclair (2008) mencionan que este tumor presenta diferenciación ductal y glandular sin el aspecto histomorfológico típico de otros carcinomas salivales con patrón especial. Además, su gran variabilidad morfológica dificulta establecer su frecuencia y el comportamiento biológico esperado.

En los archivos del Armes Forces Institute of Pathology de Estados Unidos (AFIP), revisados desde 1970, el adenocarcinoma NOS aparece tercero en frecuencia después del carcinoma mucoepidermoide y el carcinoma de células acinares; comprende alrededor del $9 \%$ de todos los tumores salivales y el $16 \%$ de los malignos. La edad promedio de aparición es de 59 años, con un rango de 3 a 94 años y un predominio en hombres (Ellis \& Auclair). Aproximadamente un 50 $\%$ de los tumores se localiza en la glándula parótida y un $40 \%$ en glándulas salivales menores, principalmente en el paladar. Se han descripto casos con otras localizaciones, como el seno maxilar, el dorso de la lengua, labios, mejilla, piso de la boca, orofaringe y área retromolar (Santoro et al., 2011; Market Velker et al., 2012; Chen et al., 2013).

Evoluciona generalmente como una masa asintomática solitaria, ocasionalmente dolorosa. A nivel del paladar son comunes su ulceración y la erosión ósea (Li et al., 2004; Ellis \& Auclair; El-Naggar et al.).

Por otro lado, Ki67 es un marcador de proliferación celular que tiene utilidad como factor pronóstico de las neoplasias. Su expresión evaluada por inmunohistoquímica se considera como una prueba de referencia con un punto de corte entre el 10 y $14 \%$ de núcleos de células neoplásicas positivamente marcadas (Li et al., 2015).

En cuanto a MUC1, es una mucina humana asociada a membrana que en las neoplasias malignas se sobre-expresa y pierde su localización exclusivamente apical (Syrkina et al., 2019).

El objetivo del presente estudio fue presentar dos casos de adenocarcinoma NOS diagnosticados por medio de la evaluación histopatológica y correlacionar la expresión de Ki67 y la localización y sobre-expresión de MUC1 con su pronóstico y grado histológico tumoral.

\section{MATERIAL Y MÉTODO}

El estudio incluyó dos casos de adenocarcinoma NOS seleccionados de la casuística de biopsias de tumores de glándulas salivales de nuestro equipo de trabajo, correspondiente al periodo 1997-2020. Se utilizó un código numérico para identificar las muestras, preservando la identidad de los pacientes.

Este trabajo es parte del proyecto "La matriz extracelular y el componente mioepitelial de los tumores epiteliales de glándulas salivales humanas: estudio estructural, histoquímico e inmunohistoquímico" aprobado por el Comité de Ética del Hospital Nacional de Clínicas. Universidad Nacional de Córdoba. Argentina (Registro 188/14). Subsidio Secretaría de Ciencia y Tecnología. Universidad Nacional de Córdoba. Argentina.

Se realizaron cortes histológicos del material incluido en bloques de parafina para su evaluación histológica con H/E. El diagnóstico morfológico de los tumores se basó en la última clasificación de la Organización Mundial de la Salud (El Naggar et al.). Después de la confirmación del diagnóstico previo por dos patólogos de manera independiente, cortes histológicos de $4 \mu \mathrm{m}$ de espesor fueron inmunomarcados con los anticuerpos monoclonales Ki67 y MUC1, con el kit LSAB+ de DAKO (Samar et al., 2004).

Ki67 detecta un antígeno nuclear cuya expresión proporciona una medida directa de la fracción de crecimiento tisular. La evaluación de la inmunomarcación se realizó por el contaje celular en campos de mayor aumento sin áreas de necrosis y homogéneas en la población celular a estudiar. Sobre cada campo se contabilizó el número total de células y las células marcadas con Ki67 y se aplicó la fórmula células marcadas/células totales por 100 para obtener el índice de proliferación. Siguiendo el St Gallen Consensus de 2009 se clasificaron los tumores como de baja, intermedia y alta proliferación de acuerdo al índice de marcación de Ki67 de $\leq 15 \%, 16-30 \%$ y $>30 \%$, respectivamente (Li et al., 2015). 
MUC-1 es un glucoproteína que se expresa en la membrana celular de los epiteliocitos orales y ductales humanos. En las células normales la expresión ocurre sólo en la membrana apical. Para el análisis comparativo, los tumores que mostraron inmunomarcación positiva en $>10 \%$ de las células se consideraron positivos. Para la evaluación semicuantitativa se clasificó la marcación en predominantemente membranosa (apical o circunferencial) o predominantemente difusa, con inmunorreactividad citoplasmática (Langner et al., 2004).

\section{RESULTADOS}

\section{A. Casos clínicos}

Caso 1: Mujer de 62 años que en el año 2012 consultó por una tumoración en la región parotídea izquierda, de varios meses de evolución. El tumor fue removido por parotidectomía. Se diagnosticó adenocarcinoma NOS.

Se le aplicó radioterapia post operatoria en el Instituto de Radioterapia Zunino. Fundación Marie Curie, de la ciudad de Córdoba. En diciembre de 2013 se le realizó una tomografía por emisión de positrones (PET) con resultado libre de enfermedad.

En el año 2015 comienza con molestias en la región izquierda de la base del cráneo, acúfenos, hipoacusia y fístula mastoidea con supuración. En la TAC se observó una lesión polilobulada irregular a nivel del peñasco izquierdo que ocupaba el meso y epitímpano, provocando lisis del hueso temporal y de la cavidad glenoidea. Destruía las celdillas del peñasco con compromiso del meato auditivo externo. La biopsia indicó una recidiva del adenocarcinoma de parótida. Fue derivada al Hospital Italiano de Buenos Aires donde en febrero de 2016 se le realizó una temporalectomía en la Unidad de Planificación Virtual y Navegación Intraoperatoria, la que se completó con reconstrucción con colgajos musculares, conservando la arteria carótida. La recidiva ocupaba gran parte del temporal y estaba en contacto con el seno venoso lateral y el trayecto intrapetroso de la carótida externa.

En abril de 2016 fue derivada nuevamente al Instituto de Radioterapia Zunino. Fundación Marie Curie para un tratamiento con radioterapia de intensidad modulada (IMRT) por 30 fracciones más dos ciclos de quimioterapia con cisplatino.
En la TAC de tórax se descartó un compromiso pulmonar. En el centellograma óseo se observó una hipercaptación a nivel del peñasco izquierdo y lesiones de tipo benigna a nivel de maxilar y mandíbula, columna cervical, dorsal y lumbar.

Actualmente continúa con controles periódicos en el Instituto Privado de Radioterapia Zunino y en el Hospital Italiano de Buenos Aires, permaneciendo sin recidivas.

Caso 2: Paciente mujer de 63 años que en julio de 2015 notó después de un tratamiento odontológico una molestia y un nódulo en la región parotídea izquierda y un pequeño nodo linfoide homolateral.

En febrero de 2016 consultó con un especialista de Cabeza y Cuello por dolor en el oído izquierdo. Se le solicitó una ecografía orientándose el diagnóstico hacia un tumor. En la ecografía se observó un aumento de tamaño de la glándula parótida izquierda y nodos linfoides locales y submandibulares izquierdos. Se le realizó una resonancia magnética que permitió diagnosticar en la región parotídea izquierda un proceso expansivo tumoral de $38 \mathrm{~mm}$, acompañado de imágenes periféricas de nodos linfoides. La masa invadía los planos musculares y adiposos adyacentes sin compromiso de estructuras vasculares.

En marzo de 2016 se le disecaron sólo los nodos linfoides pensando en controlar el tumor primario con radioterapia. En la disección nodal homolateral se detectaron once nodos linfoides, tres de ellos con metástasis. Se encontró además un nodo con metástasis en la cadena espinal y una pequeña parte de la parótida comprometida.

En mayo se decidió una segunda cirugía donde se efectuó la resección total de la glándula parótida con el tumor, con márgenes comprometidos. El resultado de la biopsia fue adenocarcinoma NOS.

Se indicó un plan de tratamiento con radioterapia de intensidad modulada (IMRT) ingresando al Instituto de Radioterapia Zunino. Fundación Marie Curie en junio de 2016. Luego del tratamiento quedó bajo control hasta que en agosto de 2017 se le realizó una TAC de cuello donde se observó una masa nodular de $21 \mathrm{~mm}$ de diámetro, con un centro hipodenso, sugestivo de recidiva tumoral. Dicha lesión desplazaba anteriormente a los vasos carotídeos. Había pérdida de los planos adiposos de clivaje de la región por cambios postquirúrgicos, no pudiéndose evaluar 
adenopatía en dicha región. Se realizó una interconsulta con ORL donde se consideró que era una recidiva en Nivel II de cuello izquierdo, siendo nuevamente derivada al Instituto de Radioterapia por considerarse inoperable debido a la falta del plano de clivaje con la arteria carótida izquierda.

En octubre de 2017 inició un nuevo tratamiento radiante con IMRT por 20 sesiones, más quimioterapia con cisplatino semanal en forma concomitante.

En agosto de 2018 se realizó una TAC de senos paranasales, peñasco y cuello por episodios de supuración de oído izquierdo, con molestia leves. Se observó la ocupación completa del meato auditivo externo, el oído medio y la totalidad de las celdillas mastoideas del lado izquierdo, con huesecillos no comprometidos. Se operó a la paciente y la biopsia del meato auditivo externo informó una neoplasia epitelial maligna poco diferenciada.

Se consultó con el Comité de Tumores quien recomendó realizar una radiocirugía estereotáctica (SRS) de la lesión en meato auditivo externo y celdillas mastoideas, firmando la paciente el consentimiento.

En setiembre de 2018 finalizó la SRS. La paciente refirió una leve mejoría del dolor en el oído izquierdo pero en octubre comienza de nuevo con episodios de dolor intenso en el oído y la zona temporal, irradiado al ojo y mandíbula homolaterales.

En la resonancia magnética realizada en noviembre se encontró una lesión hiperintensa e irregular en la punta del peñasco izquierdo, que comprometía la duramadre de la base de la fosa craneal media. Se diagnosticó una osteomastoiditis izquierda y ocupación del meato auditivo externo por lo que se derivó a la paciente a un especialista del dolor.

En mayo de 2019 se le realizó quimioterapia con criterio paliativo.

\section{B. Informe Anátomo-Patológico (Hematoxilina/ eosina) e inmunohistoquímico (Ki67 y MUC-1) de los casos clínicos}

\section{Caso 1.}

\section{Macroscopía}

1.Tejido periparotídeo izquierdo. Se recibió un fragmento de tejido pardo blanquecino translúcido indurado en el cual se reconoció un vaso sanguíneo de $24 \times 6 \mathrm{~mm}$.

2. Tejido celular subcutáneo cervical izquierdo. Se recibieron tres fragmentos de tejido adiposo, se aislaron de ellos tres estructuras nodulares de color pardo vinculables a nodos linfoides.

3. Tejido profundo. Se recibió un fragmento de tejido pardo negruzco de $4 \times 2 \mathrm{~mm}$.

4.Glándula parótida izquierda. Fragmento de tejido de $65 \times 40 \mathrm{~mm}$ de superficie lisa regular, pardo grisácea; por su otra cara era de aspecto irregular y presentaba abundante tejido adiposo. Se realizaron cortes seriados y paralelos observándose una lesión pardo-blanquecina, de $35 \mathrm{~mm}$ de diámetro mayor en la porción central, de consistencia indurada, cercana a uno de los márgenes de la pieza.

\section{Microscopía}

1. Los cortes mostraron tejido conectivo fibroso infiltrado por una neoplasia epitelial maligna de aspecto glandular. Las células presentaban anisocitosis, anisocariosis e hipercromasia (adenocarcinoma poco diferenciado). Se observaron áreas de invasión perineural y una importante reacción fibrosa desmoplásica.

2.En los cortes se identificaron tres nodos linfoides con congestión de los sinusoides y reactividad de sus centros germinativos, libres de tumor.

3.Los cortes mostraron fragmentos de tejido fibroadiposo libre de tumor, con células maduras sin atipia.

4. Los cortes de esta muestra presentaron una neoplasia epitelial maligna escasamente circunscripta, donde predominaban las estructuras ductales de distinto tamaño, varias con dilatación quística, rodeadas de un estroma fibroso y abundante tejido adiposo (Fig. 1A). El epitelio ductal era acidófilo, algunos biestratificados y con importantes vacuolas intracitoplamáticas (Fig. 1B). Las formaciones glandulares adoptaban en ocasiones un patrón cribiforme, con células claras y otras acidófilas, anaplásicas (Fig. 2A). Se identificaron además nidos celulares y cordones sólidos ramificados. El tumor se consideró como de alto grado de malignidad con algunas áreas de manifiesta anaplasia y zonas de comedonecrosis (Fig. 2B). La lesión comprometía uno de los márgenes quirúrgicos y presentó invasión perineural extensa. Se acompañaba de reacción desmoplásica e infiltrado inflamatorio, con escaso tejido parotídeo remanente conservado. 


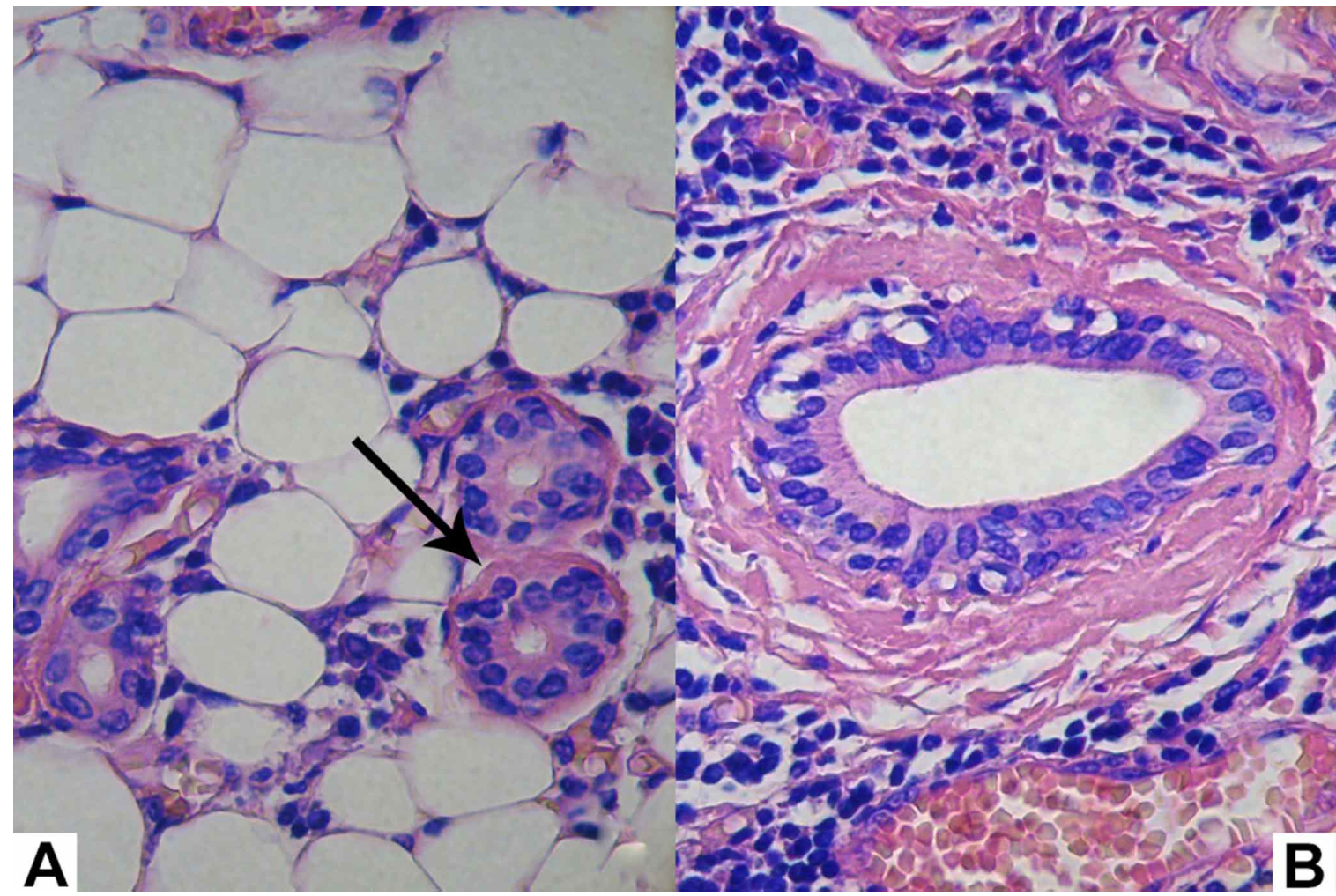

Fig. 1. Adenocarcinoma NOS. Caso 1.A. Se señalan (flecha) estructuras ductales rodeadas de tejido adiposo. H/E. 400x.B. Epitelio ductal biestratificado con citoplasma acidófilo y vacuolas intracitoplasmáticas. H/E. 400x.

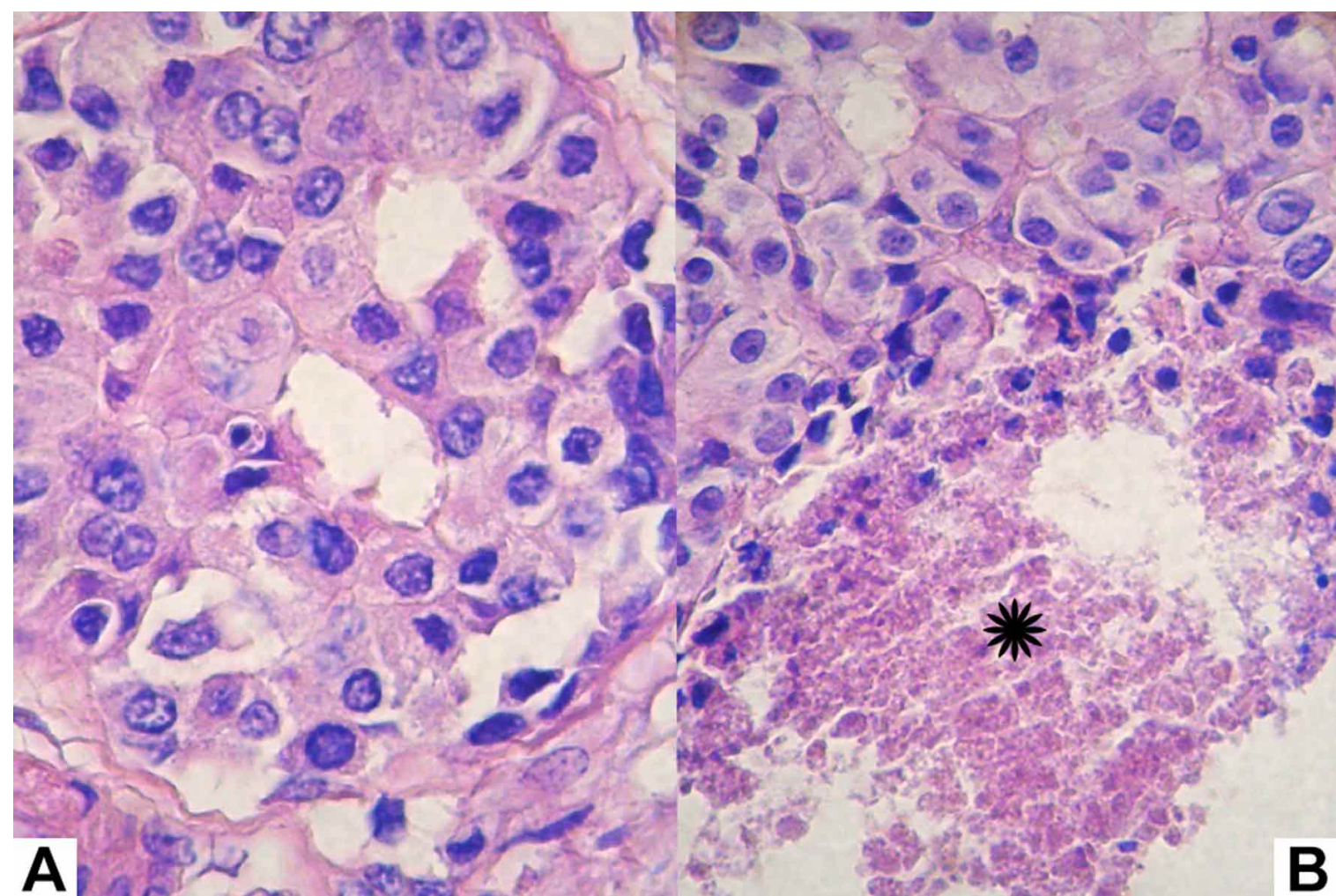

Fig. 2. Adenocarcinoma NOS. Caso 1A. Formación glandular con células anaplásicas de citoplasma acidófilo. H/E. 400x.B. Comedonecrosis de localización luminal (asterisco). H/E. 400x. 
SAMAR, R. M. E.; ÁVILA, U. R. E.; GARCíA, E. P. E.; FONSECA, A. I. B. \& FERNÁNDEZ, C. J. E. Expresión de Ki67 y MUC-1 en el adenocarcinoma no especificado de otra manera (nos) de glándulas salivales: su valor pronóstico. Int. J. Odontostomat., 14(3):407-416, 2020.

\section{Caso 2}

\section{Macroscopía}

1. Tumor maligno de parótida: se recibió un fragmento irregular de tejido, de $60 \times 35 \times 30 \mathrm{~mm}$, que mostraba una superficie externa irregular, pardo grisácea, con áreas pardo blanquecinas y que a los cortes seriados paralelos también mostraban un aspecto heterogéneo con áreas pardo blanquecinas y pardo amarillentas, de aspecto adiposo.

2. Ampliación del margen profundo: fragmento irregu-

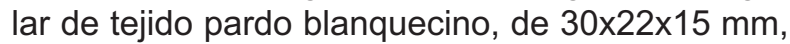
sin identificar el margen de contacto con el tumor. A los cortes seriados y paralelos mostró una coloración parda blanquecina, de aspecto fibroso.

\section{Microscopía.}

1 y 2- En los cortes se observó glándula parótida con una extensa infiltración de células anaplásicas, más importante que en el Caso 1, con un patrón de disposición en nidos o difusas (Fig. 3A). Las estructu- ras ductales presentaron similares características que el Caso 1, pero en menor cantidad (Fig. 3B). El estroma era de tipo desmoplásico, con infiltrado inflamatorio y áreas hemorrágicas. Había permeación vascular e infiltración perineural con compromiso de los márgenes de resección y del tejido muscular, con disrupción de fibras (Fig. 3C). En uno de los sectores se destacaba un conducto con displasia epitelial. Este tumor se diagnosticó como de alto grado de malignidad.

En ambos casos la marcación con Ki67 y MUC1 (Tabla I) fue bien manifiesta e intensa (Figs. 4A, B, C y Figs. 5A y B).

\section{Indice de marcación de Ki 67 (antígeno nuclear aso- ciado a proliferación celular)}

$\leq 15 \%$ : baja proliferación

16-30\%: proliferación intermedia

$>30 \%$ : proliferación alta

\section{Marcación de MUC-1}

$+:>10 \%$ de células marcadas

Predominantemente difusa: membrana y citoplasma

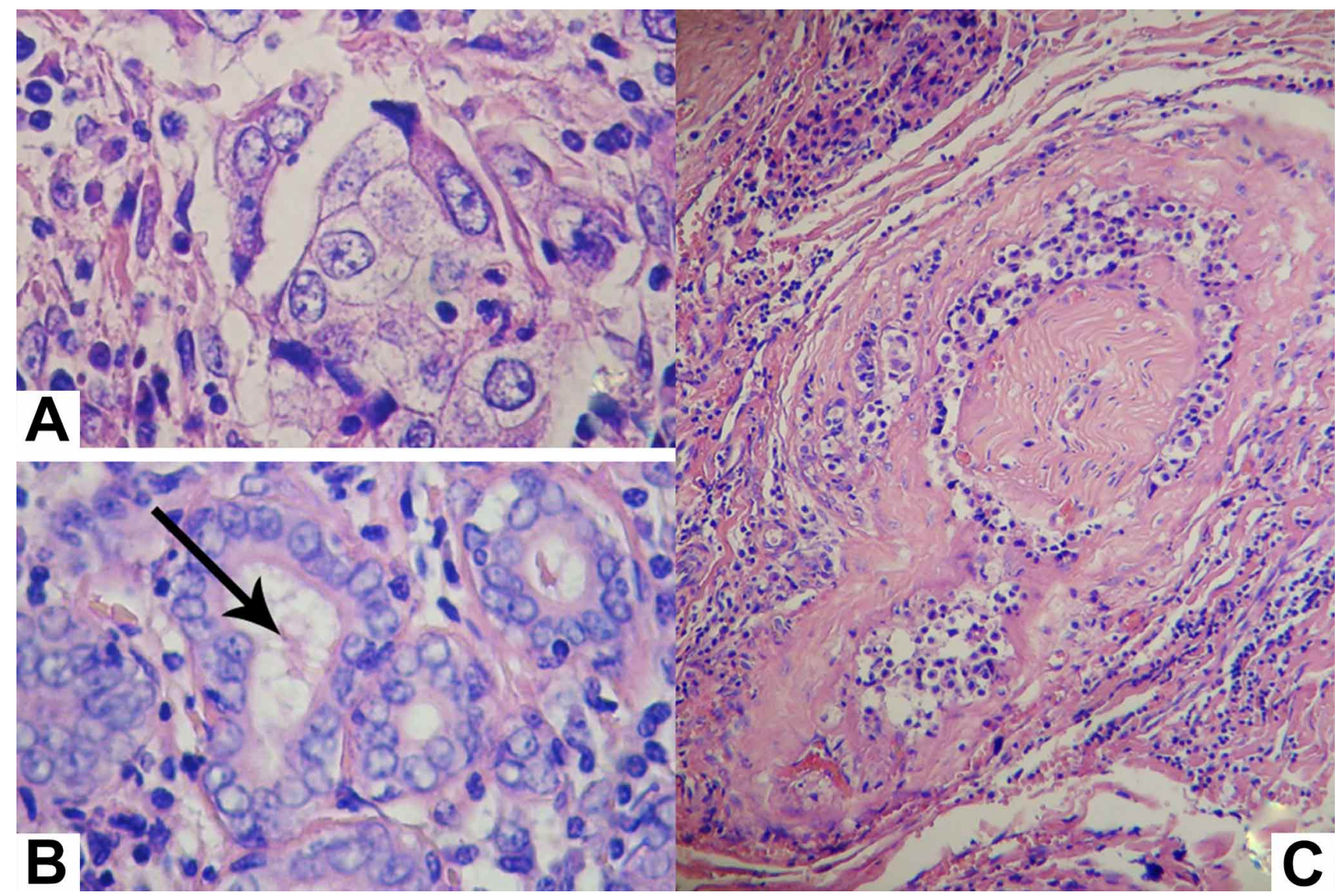

Fig. 3. Adenocarcinoma NOS. Caso 2 A. Células anaplásicas. H/E. 400x. B. Formaciones ductales con células de citoplasma acidófilo y contenido luminal (flecha). H/E. 400x. C. Infiltración perineural. H/E. 100x. 


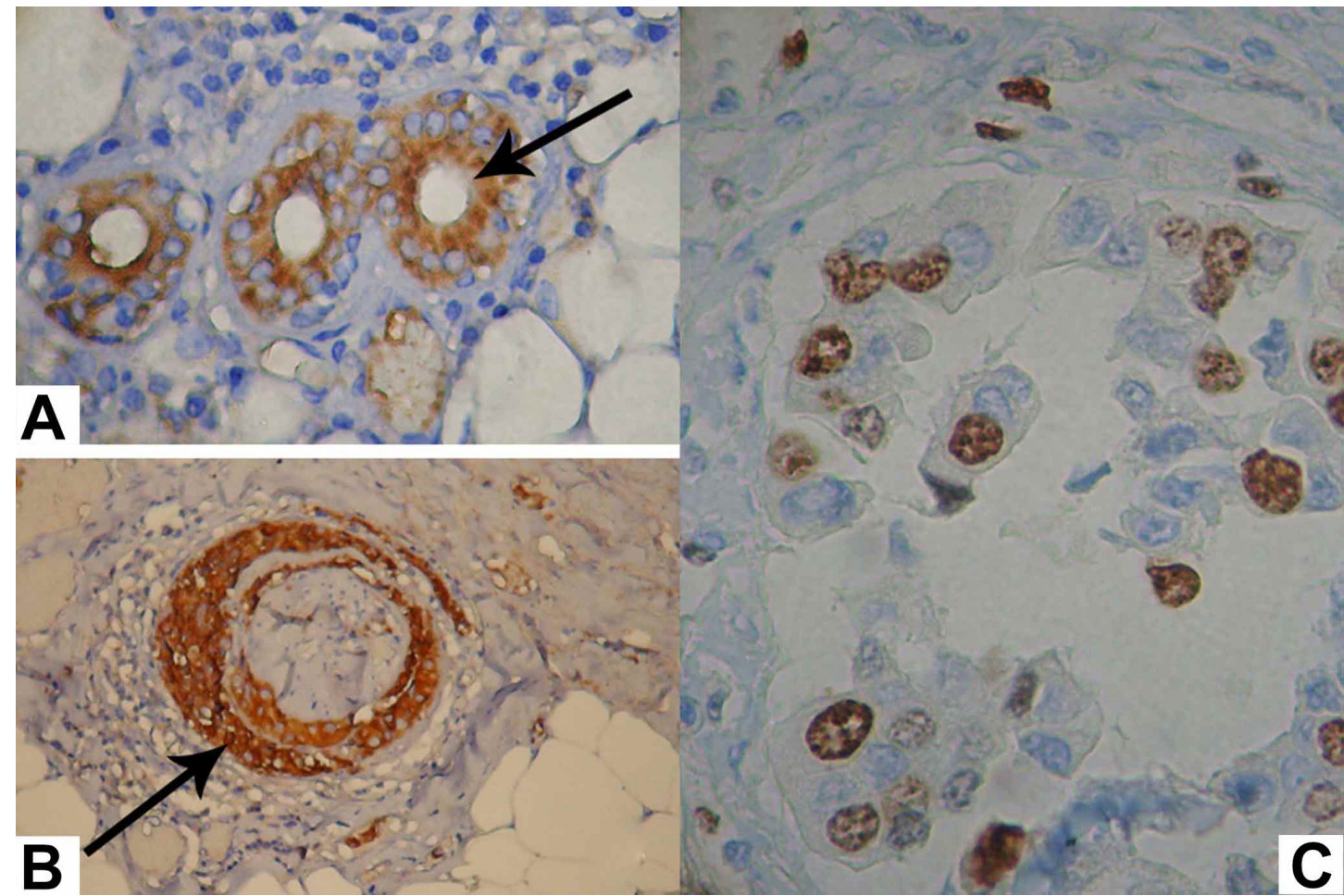

Fig. 4. Adenocarcinoma NOS. Caso 1. A. Se señalan (flecha) estructuras ductales con marcación de MUC-1 predominante difusa (membrana y citoplasma). Inmunohistoquímica MUC-1. 400x. B. Invasión perineural. Células tumorales con marcación MUC-1 difusa (flecha). Inmunohistoquímica MUC-1. 100x. C. Células tumorales con núcleos Ki67 positivos. Inmunohistoquímica Ki67. 400x.

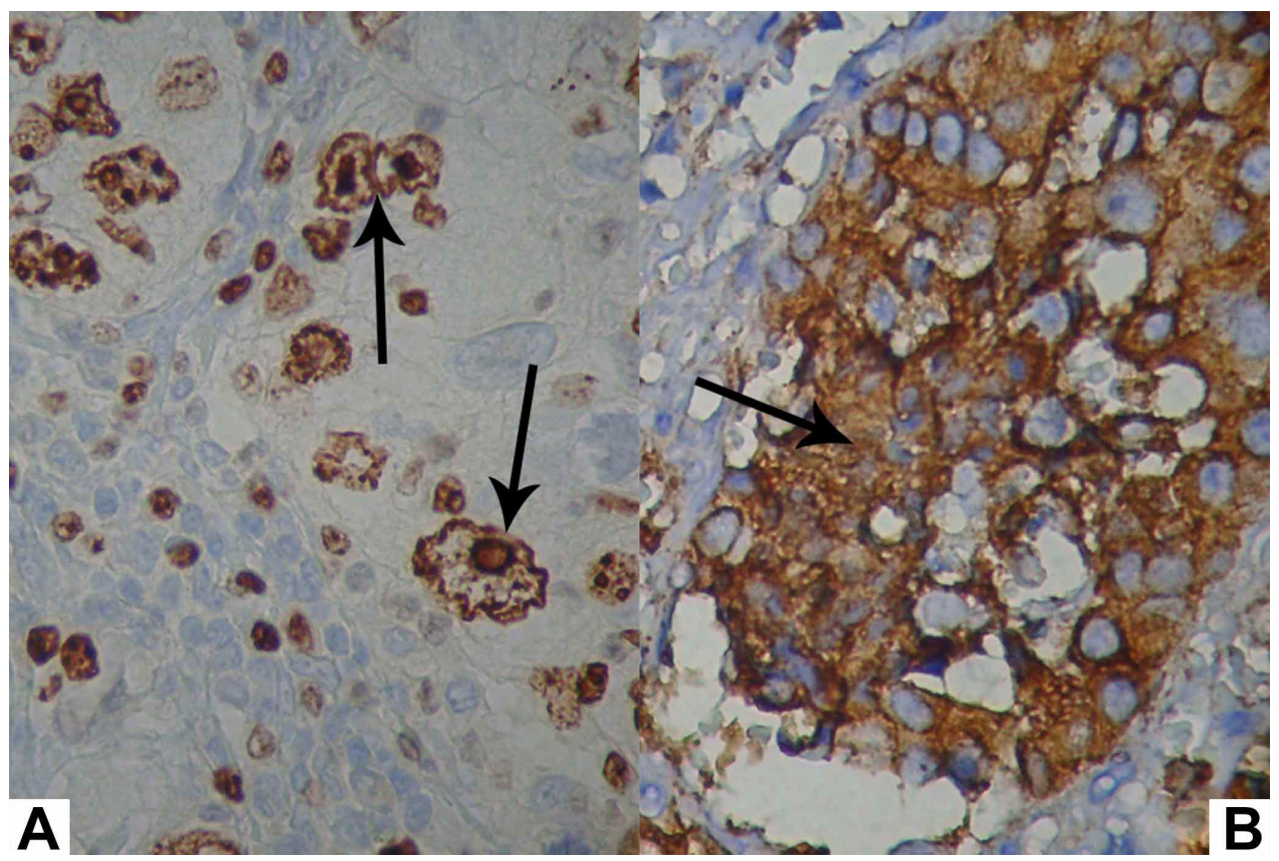

Fig. 5. Adenocarcinoma NOS. Caso 2. A. Células anaplásicas fuertemente positivas para Ki67 (flechas). Inmunohistoquímica Ki67. 400x. B. Células tumorales con sobre-expresión de MUC-1 (flecha). Inmunohistoquímica MUC1. $400 \mathrm{x}$

Tabla I. Adenocarcinoma NOS. Análisis inmunohistoquímico.

\begin{tabular}{lll}
\hline CASO & \multicolumn{1}{c}{ Ki 67} & MUC-1 \\
\hline 1 & $20 \%$ (proliferación intermedia) & + Predominantemente difusa \\
2 & $35 \%$ (alta proliferación) & + Predominantemente difusa \\
\hline
\end{tabular}


SAMAR, R. M. E.; ÁVILA, U. R. E.; GARCíA, E. P. E.; FONSECA, A. I. B. \& FERNÁNDEZ, C. J. E. Expresión de Ki67 y MUC-1 en el adenocarcinoma no especificado de otra manera (nos) de glándulas salivales: su valor pronóstico. Int. J. Odontostomat., 14(3):407-416, 2020.

\section{DISCUSIÓN}

En 1982, Spiro et al. (1982) publicaron un estudio clinicopatológico de 204 pacientes con diagnóstico de adenocarcinoma de origen salival y consideraron que muchos de los carcinomas originados en las glándulas salivales no se pueden clasificar con claridad en categorías y subclases por lo que se los menciona sólo como adenocarcinomas.

Posteriormente, en 1988, Matsuba et al. (1988) estudiaron 54 casos de adenocarcinomas localizados en las glándulas salivales mayores y menores, a los que clasificaron en adenocarcinomas bien diferenciados, moderadamente diferenciados y poco diferenciados. Estos investigadores estimaron que por el escaso número de casos presentados en los diferentes trabajos donde se clasifica a estos tumores, no se debía emplear todavía en la clínica esa clasificación.

Batsakis et al. (1992), consideraron que la mayor precisión en la clasificación tumoral basada en una correlación clínico-patológica, llevó a una reducción de la casuística del adenocarcinoma NOS (not otherwise specified).

La Organización Mundial de la Salud en su clasificación del año 1972 lo menciona sólo con el término adenocarcinoma y es partir de su $2^{\circ}$ edición 1992 que introduce la denominación adenocarcinoma not otherwise specified (NOS) (Li et al., 2004). El diagnóstico de nuevos tipos de tumores salivales que se incluyen en la $3^{\circ}$ edición 2005 de la Organización Mundial de la Salud (Barnes et al., 2005) ha disminuido de manera importante la frecuencia de este adenocarcinoma sin patrón especial.

$\mathrm{Li}$ et al. (2004) recomiendan que los adenocarcinomas NOS de alto grado se deben diferenciar de los de bajo grado por la presencia de una o más de estas características histopatológicas: atipia nuclear, alto índice mitótico, mitosis atípicas, necrosis, invasión perineural, invasión ósea, invasión vascular y un patrón de invasión agresivo.

Basándonos en estas recomendaciones hemos considerado que los dos casos por nosotros estudiados corresponden a adenocarcinomas NOS de alto grado.

En el Caso 1 se observó atipia celular, comedonecrosis, invasión perineural, compromiso de los márgenes quirúrgicos e infiltración del tejido periparotídeo. En el Caso 2 el crecimiento era infiltrante, con compromiso de los márgenes quirúrgicos; atipia celular, áreas hemorrágicas, permeación vascular e infiltración perineural y compromiso del tejido muscular. Además de estas características histopatológicas ambos casos presentaron una importante marcación positiva con Ki67 y MUC-1.

Como bien se describe en la literatura, Ki67 es una proteína marcadora de multiplicación celular cuya positividad se asocia con la proliferación e invasión de las neoplasias, aportando información sobre la agresividad de un tumor (Jing et al., 2019).

Esta proteína nuclear se expresa en las células durante las fases activas de su ciclo (G1, S, G2 y M) y está ausente en las que se encuentran en estado de reposo (G0); a diferencia del índice mitótico, que solo evidencia división celular actual, Ki67 es un fiel marcador de la actividad proliferativa, la que se relaciona con el grado tumoral y por lo tanto tiene utilidad como factor pronóstico (Cheuk \& Chan, 2007; Bussari et al., 2018).

La inmunohistoquímica es una herramienta eficaz para el diagnóstico histopatológico en la mayoría de los casos cuando existen dudas, y ayuda en el establecimiento del diagnóstico definitivo o en la confirmación de lo observado en cortes histológicos coloreados con Hematoxilina y Eosina. El anticuerpo contra el antígeno Ki67 se ha utilizado como un medio simple, rápido y confiable para evaluar la fracción de crecimiento de las poblaciones de células normales y neoplásicas (Bussari et al).

Además, según algunos estudios, Ki67 no solo es un marcador útil para evaluar el potencial proliferativo de los tumores, sino que también se puede usar como una herramienta de diagnóstico confiable que contribuye a diferenciar los subtipos de ciertos tumores malignos y a su clasificación, como el carcinoma mucoepidermoide, el carcinoma adenoide quístico y el carcinoma de células acinares, que generalmente resultan difíciles de diagnosticar solo con criterios histopatológicos (Bussari et al).

La inmunoreactividad de Ki67 ha sido considerada en numerosos estudios de cáncer como de valor tanto diagnóstico como pronóstico, principalmente en cáncer de mama, donde actualmente constituye uno de los estudios iniciales básicos junto a los receptores hormonales y Her2, para poder realizar la nueva clasificación molecular lo que orienta y define qué tratamiento se va a indicar (Pathmanathan \& Balleine, 2013). 
Numerosos estudios señalan que la proliferación celular es mayor en los tumores malignos de glándulas salivales que en los benignos, lo que demuestra su utilidad en el diagnóstico diferencial y en el pronóstico de varios subtipos histológicos, donde se relaciona directamente el porcentaje de Ki67 con su grado histológico tumoral (Vacchi-Suzzi et al., 2010).

Por otro lado, el mucus, fluido acuoso complejo cuyo principal componente son las glucoproteínas (mucinas), protege las superficies mucosas de la desecación, la injuria mecánica y la agresión bacteriana (Liu et al., 2002; Bansil \& Turner, 2018).

Las mucinas se diferencian en dos subfamilias según su localización en la superficie celular: mucinas secretadas y mucinas transmembrana o unidas a membrana.

Entre las mucinas transmembrana se encuentra MUC-1, mucina que se descubrió hace unos 40 años dentro de los glóbulos de grasa de la leche (Hattrup \& Gendler, 2008; Syrkina et al.).

La mucina humana MUC-1 fue modificando su nombre con el tiempo: antígeno epitelial de membrana (EMA), PAS-O, antígeno DF3, antígeno $\mathrm{H} 23$, antígeno NCR11, PEM, mucina urinaria unida a la lectina del maní, antígeno del suero mamario (MSA), etc. (Syrkina et al.).

En las glándulas salivales normales esta mucina se expresa en la superficie apical de la membrana de los epiteliocitos secretores y de las células de los conductos intercalados y estriados (Ponce-Bravo et al., 2008).

Por el contrario, la MUC-1 asociada al cáncer de glándulas salivales presenta una glicosilación aberrante y una expresión abundante y una pérdida de la localización apical, ocupando toda la superficie de la membrana, el citoplasma de las células tumorales y, a veces, el núcleo celular como se demostró en 40 pacientes con carcinoma de células acinares de glándulas salivales (Syrkina et al.; Ma et al., 2019).

MUC-1 inhibe las interacciones célula/célula, célula/estroma, alterando la adhesión celular y facilitando la metástasis y la progresión tumoral (Karaulov et al., 2016).

En la literatura se encuentran numerosos trabajos de la expresión de estas mucinas en distintos tipos de cáncer (Lau et al., 2004). Por el contrario, las inves- tigaciones a nivel de las neoplasias salivales son escasas (Hamada et al., 2004; Siyi et al., 2014; Barbosa Ferreira et al., 2014) y se realizaron en el carcinoma mucoepidermoide, el carcinoma de células acinares y el adenoma pleomórfico.

Y en relación a MUC-1/Ki67 solo encontramos una publicación donde Gonçalves et al. (2011) comparan su inmunoexpresión y los datos clinicopatológicos en el carcinoma mucoepidermoide de pacientes jóvenes y adultos.

Nuestros resultados demuestran que en el adenocarcinoma NOS, tumor salival sin patrón histológico especial, existe una mayor actividad proliferativa demostrada con Ki67 y sobre-expresión de MUC-1, que se asocian a atipia celular manifiesta, con infiltración perineural, necrosis y compromiso de los márgenes quirúrgicos, factores asociados a un peor pronóstico. El reconocimiento de este tumor es trascendente para el médico y el odontólogo ya que debido a la ausencia de rasgos distintivos que sí presentan otros carcinomas más definidos es fundamental el diagnóstico de exclusión.

SAMAR, R. M. E.; ÁVILA, U. R. E.; GARCÍA, E. P. E.; FONSECA, A. I. B. \& FERNÁNDEZ, C. J. E. Ki67 and MUC-1 expression in adenocarcinoma not otherwise specified (NOS) of salivary glands: prognostic value. Int. J. Odontostomat., 14(3):407-416, 2020.

ABSTRACT: Adenocarcinoma NOS (not otherwise specified) is a no special pattern salivary tumor briefly mentioned in the literature; its diagnosis is a challenge because structurally it is not identified with other more definite salivary carcinomas. On the other hand, Ki67 is a marker of cellular proliferation that provides prognostic information of neoplasms. As for human transmembrane mucin, MUC-1 is overexpressed in malignant neoplasms, losing their exclusively apical location. We present two cases of adenocarcinoma NOS diagnosed with $\mathrm{H} / \mathrm{E}$ and correlate the expression of $\mathrm{Ki} 67$ and the location and over-expression of MUC-1 with its histological grade and prognosis. Histological sections of two NOS adenocarcinomas of parotid in women of 62 and 63 ages respectively were stained with $\mathrm{H} / \mathrm{E}$ and immunolabelled for Ki67 and MUC-1. Both are predominated by ductal structures, some cystic, branched cell cords and solid islets. The glandular formations presented clear cells and some of oncocytic appearance. There was important cellular atypia, comedonecrosis, perineural growth, haemorrhagic areas and compromise of surgical margins. Nuclear marking with Ki67 was important; MUC-1 presented a strong staining in membranes and cytoplasms. They were diagnosed as high-grade malignancy. Our results show that there is an important proliferation marked with Ki67 and overexpression of MUC-1 associated with cellular atypia, perineural growth, necrosis and compromise of surgical margins, factors 
SAMAR, R. M. E.; ÁVILA, U. R. E.; GARCíA, E. P. E.; FONSECA, A. I. B. \& FERNÁNDEZ, C. J. E. Expresión de Ki67 y MUC-1 en el adenocarcinoma no especificado de otra manera (nos) de glándulas salivales: su valor pronóstico. Int. J. Odontostomat., 14(3):407-416, 2020.

associated with a poor prognosis. The recognition of this tumor is transcendent for physicians and dentists since, due to the absence of distinctive features that other more specific carcinomas present, the diagnosis of exclusion is essential.

MUC-1, prognosis.

KEY WORDS: parotid, adenocarcinoma NOS, Ki67,

\section{REFERENCIAS BIBLIOGRÁFICAS}

Bansil, R. \& Turner, B. S. The biology of mucus: Composition, synthesis and organization. Adv. Drug Deliv. Rev., 124:3-15, 2018.

Barbosa Ferreira, J. C.; Morais, M. O.; Elias, M. R.; Batista, A. C.; Leles, C. R. \& Mendonça, E. F. Pleomorphic adenoma of oral minor salivary glands: An investigation of its neoplastic potential based on apoptosis, mucosecretory activity and cellular proliferation. Arch. Oral Biol., 59(6):578-85, 2014.

Barnes, L.; Eveson, J. W.; Reichart, P. \& Sidransky, D. (Eds.). Pathology and Genetics of Head and Neck Tumours. $3^{\text {th }}$ ed. Lyon, IARC Press, 2005.

Batsakis, J. G.; El-Naggar, A. K. \& Luna, M. A. "Adenocarcinoma, not otherwise Specified": A Diminishing Group of Salivary Carcinomas. Ann. Otol. Rhinol. Laryngol., 101(1):102-4, 1992.

Bussari, S.; Ganvir, S. M.; Sarode, M.; Jeergal, P. A.; Deshmukh, A. \& Srivastava, H. Immunohistochemical detection of proliferative marker Ki-67 in benign and malignant salivary gland tumors. J. Contemp. Dent. Pract., 19(4):375-83, 2018.

Carlson, E. R. \& Schlieve, T. Salivary gland malignancies. Oral Maxillofac. Surg. Clin. North Am., 31(1):125-44, 2019.

Chen, I. L.; Chen, Y. W.; Wu, C. H.; Kao, S. Y. \& Lui, M. T. Adenocarcinoma Not otherwise specified in the maxilla mimicking a benign sinonasal cystic lesion - A case report. Taiwan J. Oral Maxillofac. Surg., 24:50-7, 2013.

Cheuk, W. \& Chan, J. K. Advances in salivary gland pathology. Histopathology, 51(1):1-20, 2007.

El-Naggar, A. K.; Chan, J. K. C.; Grandis, J. R.; Takata, T. \& Slootweg, P. J. (Eds.). World Health Organization. Classification of Head and Neck Tumours. $4^{\text {th }}$ ed. Lyon, IARC Press, 2017.

Ellis, G. L. \& Auclair, P. L. Tumors of the Salivary Glands. AFIP Atlas of Tumor Pathology Series 4. Washington D. C., Armed Forces Institute of Pathology (AFIP), 2008.

Gonçalves, C. F.; Morais, M. O.; Alencar, R. de C.; Mota, E. D.; Silva, T. A.; Batista, A. C. \& Mendonça, E. F. Expression Of Ki-67 and MUC1 In mucoepidermoid carcinomas of young and adult patients: prognostic implications. Exp. Mol. Pathol., 90(3):271-5, 2011.

Hamada, T.; Matsukita, S.; Goto, M.; Kitajima, S.; Batra, S. K.; Irimura, T.; Sueyoshi, K.; Sugihara, K. \& Yonesawa, S. Mucin expression in pleomorphic adenoma of salivary gland: a potential role for MUC1 as a marker to predict recurrence. J. Clin. Pathol., 57(8):813-21, 2004.

Hattrup, C. L. \& Gendler, S. J. Structure and function of the cell surface (tethered) mucins. Annu. Rev. Physiol., 70:431-57, 2008.

Jing, Y.; Zhou, Q.; Zhu, H.; Zhang, Y.; Song, Y.; Zhang, X.; Huang, X.; Yang, Y.; Ni, Y. \& Hu, Q. Ki-67 is an independent prognostic marker for the recurrence and relapse of oral squamous cell carcinoma. Oncol. Lett., 17(1):974-80, 2019.

Karaulov, A. V.; Gurina, N. N.; Novikov, D. V.; Formina, S. G. \& Novikov, $\mathrm{V}$. V. Role of MUC-1 expression in tumor progression. Vestn. Ross. Akad. Med. Nauk., 71(5):392-6, 2016.

Langner, C.; Ratschek, M.; Rehak, P.; Schips, L. \& Zigeuner, R. Expression of MUC1 (EMA) and E-cadherin in renal cell carcinoma: a systematic immunohistochemical analysis of 188 cases. Mod. Pathol., 17(2):180-8, 2004.
Lau, S. K.; Weiss, L. M. \& Chu, P. G. Differential expression of MUC1, MUC2, and MUC5AC in carcinomas of various sites: an immunohistochemical study. Am. J. Clin. Pathol., 122(1):61-9, 2004.

Li, J.; Wang, B. G.; Nelson, M.; Li, L.; Hu, Y.; Urken, M. L. \& BrandweinGensler, M. Salivary adenocarcinoma, not otherwise specified: a collection of orphans. Arch. Pathol. Lab. Med., 128(12):1385-94, 2004.

Li, L. T.; Jiang, G.; Chen, Q. \& Zheng, J. N. Ki67 is a promising molecular target in the diagnosis of cancer (review). Mol. Med. Rep., 11(3):1566-72, 2015.

Liu, B.; Lague, J. R.; Nunes, D. P.; Toselli, P.; Oppenheim, F. G.; Soares, R. V.; Troxler, R. F. \& Offner, G.D. Expression of membraneassociated mucins MUC1 and MUC4 in major human salivary glands. J. Histochem. Cytochem., 50(6):811-20, 2002.

Ma, S.; An, F.; Li, L. H.; Lin, Y. Y. \& Wang, J. Expression of Mucin 1 in salivary gland tumors and its correlation with clinicopathological factors. J. Biol. Regul. Homeost. Agents, 33(2):563-9, 2019.

Market Velker, B. A.; Louie, A. V.; Velker, V. M.; Kwan, K. F.; Franklin, J. H. \& Venkatesan, V. M. Adenocarcinoma not otherwise specified on dorsum of tongue: case report and literature review. Curr. Oncol., 19(5):e358-63, 2012.

Matsuba, H. M.; Mauney, M.; Simpson, J. R.; Thawley, S. E. \& Pikul, F. J. Adenocarcinomas of major and minor salivary gland origin: a histopathologic review of treatment failure patterns. Laryngoscope, 98(7):784-8, 1988

Pathmanathan, N. \& Balleine, R. L. Ki67 and proliferation in breast cancer. J. Clin. Pathol., 66(6):512-6, 2013.

Ponce-Bravo, S.; Ledesma-Montes, C. \& Morales Sánchez, I. MUC-1 mucin in normal human salivary glands detected by HMFG-1 and HMFG-2 monoclonal antibodies. APMIS, 116(2):93-8, 2008.

Samar, M. E.; Avila, R.E. \& Esteban Ruiz, F. Técnicas Histológicas. Fundamentos y Aplicaciones. Ciudad de Córdoba, SeisC, 2004.

Santoro, A.; Laino, L.; Contaldo, M.; Pannone, G.; Guida, A.; Serpico, R. \& Bufo, P. Case report. Adenocarcinoma NOS of the maxillary sinus: clinical and histopathological features with therapeutic considerations. Otolaryngol. Open Access, 1 (1)103, 2011.

Siyi, L.; Shengwen, L.; Min, R.; Wenjun, Y.; Lizheng, W. \& Chenping, $Z$. Increased expression of MUC-1 has close relation with patient survivor in high-grade salivary gland mucoepidermoid carcinoma. J. Oral Pathol. Med., 43(8):579-84, 2014.

Spiro, R. H.; Huvos, A. G. \& Strong, E. W. Adenocarcinoma of salivary origin. Clinicopathologic study of 204 patients. Am. J. Surg., 144(4):423-31, 1982.

Syrkina, M. S.; Vassetzky, Y. S. \& Rubtsov, M. A. MUC1 story: great expectations, disappointments and the renaissance. Curr. Med. Chem., 26(3):554-63, 2019.

Vacchi-Suzzi, M.; Bocciolini, C.; Bertarelli, C. \& Dall'Olio, D. Ki-67 proliferation rate as a prognostic marker in major salivary gland carcinomas. Ann. Otol. Rhinol. Laryngol., 119(10):677-83, 2010.

Direccion para correspondencia:

María Elena Samar Romani

Catamarca 1546.

Barrio General Paz

Córdoba (5000)

ARGENTINA

Email: samarcongreso@gmail.com

Recibido : 28-02-2020

Aceptado: 20-04-2020 